\title{
Antigen-Directed Cancer Surgery for Primary Colorectal Cancer: 15-Year Survival Analysis
}

\author{
J. P. Tiernan, MBChB, PhD, FRCS and D. G. Jayne, BSc, MB BCh, FRCS, MD \\ Leeds Institute of Molecular Medicine, St James's University Hospital, Leeds, UK
}

\section{TO THE EDITORS:}

We read with interest the article by Povoski et al. concerning the long-term follow-up of patients who had resections for colorectal cancer performed using antigendirected surgery. ${ }^{1}$ The authors have been at the forefront of research into radioimmunoguided surgery (RIGS) for over 25 years and are to be congratulated on their work. This paper compared survival data for 93 patients who underwent antigen-directed surgery between 1992 and 1995 and found that those who had no detectable bound antibody remaining in the surgical field at the end of the procedure had a significant survival advantage over those who did.

The difference in survival between the TAG-72-positive and TAG-negative groups reached significance only for advanced-stage (III and IV) disease and only at the 10- and 15-year intervals. In the absence of precise data describing which patients had extra tissue removed or a change in operation as a direct result of TAG-72 detection, it cannot be concluded that RIGS was the causal factor of this difference. It may simply have identified those patients with tumour cells remaining but not affected the outcome. Considering adjuvant treatment is indicated in both groups anyway, it is difficult to see the technique being adopted on a large scale based on this premise.

We also found it interesting that 21 patients with earlystage $(0$, I, or II) disease showed TAG-72 positivity yet there was no difference in survival when compared with the TAG-72-negative group, even at 15 years. Although the numbers are small, this raises concerns about the accuracy of the system, particularly as normal colon is known to express the antigen. ${ }^{2}$

We see the application of antigen-directed surgery in the modern management of colorectal cancer lying in identifying occult metastases that would indicate a need for adjuvant chemotherapy (i.e. upstaging stage II disease to stage III) or consideration for a more radical 'D3' resection, as described by Hoenberger et al. ${ }^{3}$ Unfortunately it is not possible to evaluate the feasibility of this based on the data presented. With larger numbers of stage II patients this may become clearer.

\section{REFERENCES}

1. Povoski S, et al. Antigen-directed cancer surgery for primary colorectal cancer: 15-year survival analysis. Ann. Surg. Oncol. 2012;19(1):131-8.

2. Listrom MB, et al. Immunoreactivity of tumor-associated glycoprotein (TAG-72) in normal, hyperplastic, and neoplastic colon. Human Pathol. 1989;20(10):994-1000.

3. Hohenberger W, et al. Standardized surgery for colonic cancer: complete mesocolic excision and central ligation-technical notes and outcome. Colorectal Dis. 2009;11(4):354-64.
(C) Society of Surgical Oncology 2017

First Received: 20 October 2017; Published Online: 31 October 2017

J. P. Tiernan, MBChB, PhD, FRCS

e-mail: jimtiernan1@gmail.com 